\title{
Neoadjuvant programmed death-1 blockade plus chemotherapy in locally advanced esophageal squamous cell carcinoma
}

\author{
Guozhen Yang ${ }^{1,2,3}$, Xiaodong Su ${ }^{1,2,3 \#}$, Hong Yang ${ }^{1,2,3}$, Guangyu Luo ${ }^{1,4}$, Chan Gao ${ }^{5}$, Yating Zheng ${ }^{5}$, \\ Wenzhuan Xie ${ }^{5}$, Mengli Huang ${ }^{5}$, Ting $\mathrm{Bei}^{5} \wedge$, Yuezong Bai ${ }^{5}$, Zhiqiang Wang ${ }^{1,6}$, Peiqiang Cai ${ }^{1,7}$, \\ Haoqiang He $\mathrm{He}^{1,7}$, Jin Xiang ${ }^{1,8}$, Muyan Cai ${ }^{1,8}$, Yijun Zhang ${ }^{1,8}$, Chunhua Qu ${ }^{1,8}$, Jianhua Fu ${ }^{1,2,3}$, \\ Qianwen Liu $^{1,2,3}$, Yi Hu ${ }^{1,2,3}$, Jiudi Zhong ${ }^{1,2,3}$, Yuanheng Huang ${ }^{1,2,3}$, Qiyu Guo ${ }^{1,2,3}$, Xu Zhang ${ }^{1,2,3}$
}

${ }^{1}$ State Key Laboratory of Oncology in South China, Collaborative Innovation Center of Cancer Medicine, Guangzhou, China; ${ }^{2}$ Guangdong Esophageal Cancer Institute, Guangzhou, China; ${ }^{3}$ Department of Thoracic Oncology, Sun Yat-sen University Cancer Center, Guangzhou, China; ${ }^{4}$ Department of Endoscopy, Sun Yat-sen University Cancer Center, Guangzhou, China; ${ }^{5}$ Medical Affairs, 3D Medicines, Inc., Shanghai, China; ${ }^{6}$ Department of Medical Oncology, Sun Yat-sen University Cancer Center, Guangzhou, China; ${ }^{7}$ Department of Medical Imaging and Interventional Radiology, Sun Yat-sen University Cancer Center, Guangzhou, China; ${ }^{8}$ Department of Pathology, Sun Yat-sen University Cancer Center, Guangzhou, China

Contributions: (I) Conception and design: X Zhang, G Yang; (II) Administrative support: X Zhang, G Yang; (III) Provision of study materials or patients: All authors; (IV) Collection and assembly of data: All authors; (V) Data analysis and interpretation: All authors; (VI) Manuscript writing: All authors; (VII) Final approval of manuscript: All authors.

\#These authors contributed equally to this work.

Correspondence to: Xu Zhang, MD, PhD, Department of Thoracic Oncology, State Key Laboratory of Oncology in South China, Collaborative Innovation Center of Cancer Medicine, Guangdong Esophageal Cancer Institute, Sun Yat-sen University Cancer Center, 651 Dong Feng Road East, Guangzhou 510060, China. Email: zhangxu@sysucc.org.cn.

Background: Immunotherapy is effective in treating unresectable esophageal squamous cell carcinoma (ESCC), but little is known about its role in the preoperative setting. The aim of this study was to evaluate the safety, feasibility and efficacy of neoadjuvant treatment with camrelizumab plus chemotherapy in locally advanced ESCC.

Methods: Patients diagnosed with locally advanced ESCC were retrospectively included if they had received neoadjuvant camrelizumab plus nab-paclitaxel and S1 capsule followed by radical esophagectomy between November, 2019 and June, 2020 at Sun Yat-sen University Cancer Center. Primary endpoints were safety and feasibility. In addition, pathological response and the relationship between tumor immune microenvironment (TIME)/tumor mutational burden (TMB) and treatment response were also investigated.

Results: Twelve patients were included and they all received three courses of preoperative treatment with camrelizumab plus nab-paclitaxel/S1. No grade 3 or higher toxicities occurred. No surgical delay or perioperative death was reported. Nine patients $(75 \%)$ responded to the treatment, four with a complete pathological response (pCR) and five with a major pathological response (MPR). Neither programmed death-ligand 1 (PD-L1) expression nor TMB was correlated with treatment response. TIME analysis revealed that a higher abundance of CD56dim natural killer cells was associated with better pathological response in the primary tumor, while lower density of M2-tumor-associated macrophages was associated with better pathological response in the lymph nodes (LNs).

Conclusions: Neoadjuvant camrelizumab plus nab-paclitaxel and S1 is safe, feasible and effective in locally advanced ESCC and is worth further investigation.

Keywords: Anti-programmed death-1 (PD-1); esophageal squamous cell carcinoma (ESCC); neoadjuvant therapy; tumor immune microenvironment (TIME); tumor mutational burden (TMB)

^ ORCID: 0000-0001-7481-2202. 
Submitted Jun 11, 2021. Accepted for publication Aug 05, 2021.

doi: 10.21037/atm-21-3352

View this article at: https://dx.doi.org/10.21037/atm-21-3352

\section{Introduction}

With over 500,000 new cases diagnosed annually, esophageal carcinoma ranks as the seventh most common cancer worldwide and the sixth leading cause of cancer deaths in 2018 (1). Approximately $90 \%$ of all esophageal cancers are esophageal squamous cell carcinomas (ESCCs) (2). For patients diagnosed with locally advanced ESCC, preoperative chemoradiation followed by surgery is available as the standard-of-care treatment, but recurrence still occurs in $31-39 \%$ of patients within $3-5$ years after surgery $(3,4)$. Moreover, the increased risk of perioperative toxicities, complications and mortality associated with chemoradiation makes it less appealing to a vast number of patients $(5,6)$.

In recent years, anti-programmed death-1 (PD-1)/antiprogrammed death-ligand 1 (PD-L1) has demonstrated great promise in unresectable ESCC patients, and thus has been approved by the Food and Drug Administration as second-line treatment in this population $(7,8)$. In addition, neoadjuvant administration of anti-PD-1 in other malignancies such as lung cancer, melanoma and colorectal cancer has also produced durable responses with favorable tolerability (9-11). Multiple trials are therefore currently underway to exploit preoperative use of anti-PD-1/PDL1 in locally advanced ESCC, most of which combined anti-PD-1/PD-L1 with chemotherapy or chemoradiation. However, according to the preliminary data presented by Lee et al. at ESMO Congress 2019, upon preoperative administration of pembrolizumab plus platinum-based chemoradiotherapy, eight deaths (out of 28 patients) occurred, two due to pre-surgical hematemesis, two resulting from acute lung injury, and four due to disease progression (12). Despite a $46.1 \%$ complete pathological response (pCR) rate among the patients having undergone surgery, anti-PD-1 combined with chemoradiation raised considerable safety concerns. Therefore, a less toxic regimen is needed in this scenario.

This retrospective analysis investigates the safety and feasibility of neoadjuvant treatment with camrelizumab plus nab-paclitaxel and S1 in a small cohort of patients with locally advanced ESCC. Treatment responses assessed by radiography were also evaluated in comparison with surgical pathology. Since predictive biomarkers predicting treatment response are urgently needed to be identified, the correlation between tumor immune microenvironment (TIME)/tumor mutational burden (TMB) and treatment response was also explored. We present the following article in accordance with the STROBE reporting checklist (available at https://dx.doi.org/10.21037/atm-21-3352).

\section{Methods}

\section{Patients and study design}

ESCC patients who received camrelizumab plus nabpaclitaxel and S1 before standard radical esophagectomy between November, 2019 and June, 2020 at Sun Yat-sen University Cancer Center were retrospectively screened. They were included if they had: (I) a diagnosis of stage T2-3, N0-3 locally advanced ESCC confirmed with contrast-enhanced computerized tomography (CT), EUS, and cervical lymph node (LN) ultrasonography; (II) no cervical LN metastasis or distant organ metastasis; and (III) no secondary primary tumors. All patients were subjected to post-treatment evaluation using contrast-enhanced CT and cervical LN ultrasonography within seven days before surgery. Patients' de-identified data were extracted from their electronic medical records.

The primary endpoints of the study were safety and feasibility. The secondary end points were radiographic and pathological responses. An exploratory objective of this study was to identify genomic and immunologic features that may predict benefit from neoadjuvant immunochemotherapy in locally advanced ESCC. This study was approved by the institutional review boards at Sun Yat-sen University Cancer Center (B2020-292-01) and all patients provided written informed consents. All procedures performed in this study involving human participants were in accordance with the Declaration of Helsinki (as revised in 2013).

\section{Assessment}

Treatment-related adverse events (TRAEs) were reported according to the National Cancer Institute Common 
Terminology Criteria for Adverse Events, version 5.0 (13). Feasibility was defined as no surgical delay due to immunerelated TRAEs and no severe perioperative complications. Radiographic responses of primary tumors were evaluated using CT scan images acquired before and after neoadjuvant treatment per Response Evaluation Criteria in Solid Tumors version (RECIST) 1.1 (14). Pathological regression was assessed using hematoxylin and eosin $(\mathrm{H} \& \mathrm{E})$ stained slides of surgical specimens. Tumors with $\leq 10 \%$ residual viable tumor cells were considered as having achieved a major pathological response (MPR) while those showing no residual tumor were defined as having a pCR. Patients with $\geq 50 \%$ remaining viable tumor were classified as nonresponders. All imaging data and pathological data were reviewed by two independent radiologists or pathologists.

\section{TIME}

Investigation of the TIME was performed by 3D Medicines, Inc, a College of American Pathologists (CAP)-accredited and Clinical Laboratory Improvement Amendments (CLIA)-certified laboratory. PD-L1 expression was assessed using the PD-L1 IHC 22C3 pharmDx assay (Agilent Technologies, CA, USA) and was expressed as combined positive score (CPS) by dividing the number of PD-L1stained tumor and immune cells with the total number of viable tumor cells and multiplying by 100 . Multiplex immunofluorescence (mIF) staining was conducted using the PANO 7-plex IHC kit following manufacturer's instructions (Panovue, Beijing, China). Multiplex stained slides were scanned using a Mantra system (PerkinElmer, MA, USA) configured to capture fluorescent spectra at $20 \mathrm{~nm}$ wavelength intervals from $420 \mathrm{~nm}$ to $720 \mathrm{~nm}$ with a fixed exposure time and an absolute magnification of $\times 200$ and $\times 100$. All scans for each slide were then superimposed to obtain a single image. Images of unstained and monoplex stained slides were used to extract tissue autofluorescence and the spectrum of each fluorophore, respectively. They were also used to create a spectral library required for multispectral unmixing using the inForm Image Analysis software v.2.4 (PerkinElmer, MA, USA). Slide images were reconstructed without autofluorescence using this spectral library. The quantity of CD8+ T cells, macrophages and natural killer cells were expressed as the number of stained cells per square millimeter.

\section{Next generation sequencing (NGS) and TMB determination}

NGS was performed on pretreatment tissues as described previously using the GPS panel targeting the exons of 733 selected cancer-related genes (3D Medicines Inc., Shanghai, China) (see Table S1 for a full list of the 733 genes included in the panel) (15). Briefly, genomic DNA (gDNA) was isolated from formalin-fixed paraffin-embedded (FFPE) tissue sections with a $\geq 20 \%$ tumor content using the ReliaPrep FFPE gDNA Miniprep System (Promega). DNA extracts (30-200 ng) were sheared to $250 \mathrm{bp}$ fragments using a S220 focused-ultrasonicator (Covaris, Inc., MA, USA). Libraries were prepared using the KAPA Hyper Prep Kit (KAPA Biosystems, Cape Town, South Africa) following the manufacturer's protocol, followed by probe-based hybridization with a customized NGS panel targeting exons of 733 cancer-related genes. The captured libraries were loaded onto a NovaSeq 6000 platform (Illumina, CA, USA) for $100 \mathrm{bp}$ paired-end sequencing with a mean sequencing depth of 1,000x. Somatic single nucleotide variants (SNVs) were detected using MuTect (v1.1.7) (https://github.com/ broadinstitute/mutect) and somatic insertions and deletions (indels) using Pindel (v0.2.5a8) (http://gmt.genome.wustl. edu/packages/pindel) with default parameters. Copy number variations (CNVs) were called by an in-house developed script with a cut-off of 6 copies. The TMB was defined as the number of somatic single nucleotide variations (SNVs) and insertions/deletions (indels) per megabase of coding genome sequenced. SNVs referred to synonymous \& non-synonymous mutations, stop gain/ loss, and splicing variants. Indels included both frameshift and non-frameshift insertions and deletions. Non-coding alterations were excluded from TMB calculation.

\section{Statistical analyses}

Continuous variables were compared using Mann-Whitney $\mathrm{U}$ test and categorical variables were compared using Chisquare or Fisher exact test. All reported $\mathrm{P}$ values were two-tailed. A P value of $<0.05$ is considered statistically significant. All analyses and graph generation were 
Table 1 Baseline characteristic of the patients

\begin{tabular}{|c|c|}
\hline Characteristics & No. (\%) \\
\hline \multicolumn{2}{|l|}{ Age at diagnosis, years } \\
\hline Mean $\pm S^{a}$ & $57 \pm 5.5$ \\
\hline Median [range] & $56[50-65]$ \\
\hline \multicolumn{2}{|l|}{ Gender } \\
\hline Male & $7(58.3)$ \\
\hline Female & $5(41.7)$ \\
\hline \multicolumn{2}{|l|}{ History of smoking } \\
\hline Former or current & 7 (58.3) \\
\hline Never & $5(41.7)$ \\
\hline \multicolumn{2}{|l|}{ Site of primary tumor } \\
\hline Upper thoracic & $1(8.3)$ \\
\hline Middle thoracic & $6(50.0)$ \\
\hline Lower thoracic & $5(41.7)$ \\
\hline \multicolumn{2}{|l|}{ Histologic grade } \\
\hline Well differentiated & $1(8.3)$ \\
\hline Moderately differentiated & $3(25.0)$ \\
\hline Poorly differentiated & $8(66.7)$ \\
\hline \multicolumn{2}{|l|}{ Tumor stage $^{\mathrm{b}}$} \\
\hline II & $2(16.7)$ \\
\hline III & $8(66.6)$ \\
\hline IVA & $2(16.7)$ \\
\hline \multicolumn{2}{|l|}{ PD-L1 CPS } \\
\hline$<10$ & $9(75.0)$ \\
\hline$\geq 10$ & $2(16.7)$ \\
\hline Unevaluable & $1(8.3)$ \\
\hline
\end{tabular}

${ }^{\mathrm{a}}$, standard deviation; ${ }^{\mathrm{b}}$, tumor stage was evaluated following the American Joint Committee on Cancer's (AJCC) Staging Manual, 7th edition. PD-L1, programmed death-ligand 1; CPS, combined positive score.

performed using R 3.6.0.

\section{Results}

\section{Baseline characteristics}

A total of 12 ESCC patients who agreed to receive preoperative anti-PD-1 plus nab-paclitaxel and S1 before radical esophagectomy between November, 2019 and June,
2020 were included in the study (Table 1). In all, seven were males and five were females. The median age of the cohort was 56 years (range, 50-65 years). Two patients had stage II, eight had stage III and two had stage IVA ESCC. Twothirds of the patients had poorly differentiated tumors (see Table S2 for baseline clinicophysiological characteristics of the patients). All patients completed three courses of preoperative treatment which comprised a flat dose of camrelizumab (200 mg, IVGTT) plus a single dose of nab-paclitaxel $\left(260 \mathrm{mg} / \mathrm{m}^{2}\right.$, IVGTT) on day 1 and S1 administered twice daily [ $40 \mathrm{mg}$ for body surface area (BSA) $<1.25 \mathrm{~m}^{2}, 50 \mathrm{mg}$ for BSA $\geq 1.25$ to $<1.50 \mathrm{~m}^{2}$, or $60 \mathrm{mg}$ for BSA $\geq 1.50 \mathrm{~m}^{2}$ ] on days 1 to 14 . The cycle was repeated every three weeks for three cycles.

\section{Safety and feasibility}

Neoadjuvant use of camrelizumab in combination with nabpaclitaxel and S1 did not cause any previously unreported toxicities (Table 2). All patients experienced at least one TRAE, but no grade 3 or higher events occurred. Overall, the most common grade 1-2 events included reactive capillary hemangioma of the skin $(91.7 \%)$, muscle soreness (50.0\%), limb numbness (41.7\%), and anemia (16.7\%), among which reactive capillary hemangioma was immunerelated. Toxicities such as pneumonia, myocarditis, hepatitis, nephritis, thyroiditis and hypophysitis that are usually associated with immunotherapy were not observed. All TRAEs resolved without intervention.

All patients underwent thoracoscopic esophagectomy with cervical esophagogastric anastomosis and modern two-field LN dissection as planned. The median interval between the last dose of neoadjuvant therapy and surgery was 1.2 weeks (range, 0.4-2.9 weeks) and all patients underwent standard R0 resection, which took $253.0 \pm 28.4$ minutes on average. Intraoperative bleeding volume was $50-200 \mathrm{~mL}$. No perioperative mortality was reported. Anastomotic leakage occurred in two patients 10 and 11 days after surgery, respectively, and both were healed after one week of conservative treatment. None of the patients experienced any postoperative immune-related adverse events or other complications.

\section{Clinical and pathological responses}

According to RECIST v1.1, seven (58.3\%) patients had partial response (PR) and five (41.7\%) had stable disease (SD) (Figure 1A). Surgical pathology showed a median 
Table 2 Summary of treatment related adverse events

\begin{tabular}{|c|c|c|}
\hline \multirow{2}{*}{ All events } & \multicolumn{2}{|c|}{ No. of patients (\%) } \\
\hline & Grade 1-2 & Grade 3-4 \\
\hline \multicolumn{3}{|l|}{ Gastrointestinal } \\
\hline Nausea & $1(8.3)$ & $0(0)$ \\
\hline Vomiting & $1(8.3)$ & $0(0)$ \\
\hline Diarrhea & $1(8.3)$ & $0(0)$ \\
\hline Constipation & $1(8.3)$ & $0(0)$ \\
\hline ALT/AST increase & $0(0)$ & $0(0)$ \\
\hline \multicolumn{3}{|l|}{ Hematopoietic } \\
\hline Anemia & $2(16.7)$ & $0(0)$ \\
\hline Leukopenia & $0(0)$ & $0(0)$ \\
\hline Thrombocytopenia & $0(0)$ & $0(0)$ \\
\hline \multicolumn{3}{|l|}{ Respiratory system } \\
\hline Cough & $0(0)$ & $0(0)$ \\
\hline Pneumonia & $0(0)$ & $0(0)$ \\
\hline \multicolumn{3}{|l|}{ Peripheral nerve } \\
\hline Limb numbness & $5(41.7)$ & $0(0)$ \\
\hline Muscle soreness & $6(50.0)$ & $0(0)$ \\
\hline Cardiac troponin & $0(0)$ & $0(0)$ \\
\hline \multicolumn{3}{|c|}{ Immune-related adverse events } \\
\hline $\begin{array}{l}\text { Reactive capillary } \\
\text { hemangioma }\end{array}$ & $11(91.7)$ & $0(0)$ \\
\hline Myocarditis & $0(0)$ & $0(0)$ \\
\hline Hepatitis & $0(0)$ & $0(0)$ \\
\hline Nephritis & $0(0)$ & $0(0)$ \\
\hline Pneumonia & $0(0)$ & $0(0)$ \\
\hline Hyperthyroidism & $0(0)$ & $0(0)$ \\
\hline Hypophysitis & $0(0)$ & $0(0)$ \\
\hline
\end{tabular}

All adverse events were reported according to the National Cancer Institute Common Terminology Criteria for Adverse Events, version 5.0.

tumor regression of $97 \%$ (range, 4-100\%). Four patients achieved a pCR and five cases had an MPR in the primary tumor, which were collectively defined as responders (75\%) (Figure 1B). The other three patients only displayed a regression of $30,4 \%$ and $4 \%$, respectively, and were therefore regarded as non-responders. Ten patients had down-staging in the primary tumors and nine of the 11 patients with potential $\mathrm{LN}$ involvement according to baseline CT showed LN down-staging (see Table S2, which summarizes potential baseline $\mathrm{LN}$ involvement by CT). None of the pCR patients had LN metastasis following treatment.

Five patients exhibited discordant radiographic and pathological responses. Two had SD according to presurgical CT but were found to have an MPR and a pCR, respectively. Three of the PR patients turned out to have a pCR in resected tissue. This was consistent with previous observation that evaluation per RECIST criteria may not truly reflect actual benefit from immunotherapy (9). Of note, the surgical resections of the responders showed an inflamed phenotype with a massive influx of multinucleated giant cells and lymphocytes compared to baseline biopsy samples, a phenomenon typically associated with response to immune checkpoint blockade, which may help explain the minimal tumor shrinkage or even tumor enlargement seen in the CT scans of some responders (Figure 1C). Tertiary lymphoid structures (TLSs), which predicted favorable prognosis and improved response to immunotherapy in several solid tumors, were also observed in the resected tumor of responders, corroborating the favorable responses in these patients (16-18).

\section{Tumor regression pattern induced by anti-PD-1-based neoadjuvant therapy}

Tumor regression as revealed by surgical pathology seemed to follow a sequential pattern. Non-responders still showed invasion through the mucosa into the muscularis propria or adventitia (Figure $2 A$ Right, Table 3). Among the five MPR patients, those without invasion in the muscularis were also free of tumor in the adventitia (patients 5 and 7) while those without invasion in the submucosa were also free of tumor in the muscularis and adventitia (patients 6 and 8), suggesting that regression induced by anti-PD-1-based neoadjuvant therapy may start from the outermost layer and extend towards the innermost layer of the esophageal wall (Figure 2B).

\section{Immunologic and genomic correlates of response to anti- PD-1-based neoadjuvant therapy}

In KEYNOTE-180, a CPS PD-L1 of $\geq 10$ was associated with a slight improvement of objective response rate in advanced esophageal cancer, therefore we also examined PD-L1 expression in our cohort (8). Among the 


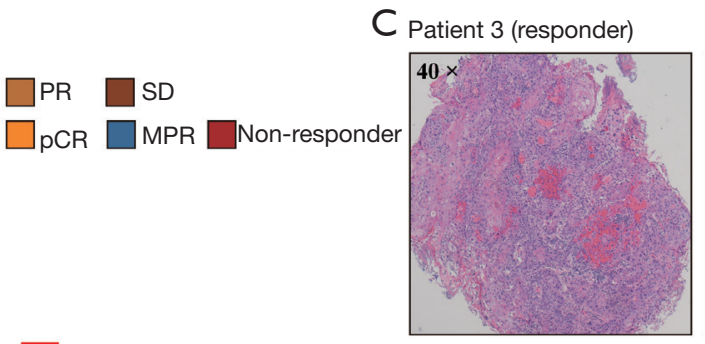

Lymph node (-)

Lymph node (+)

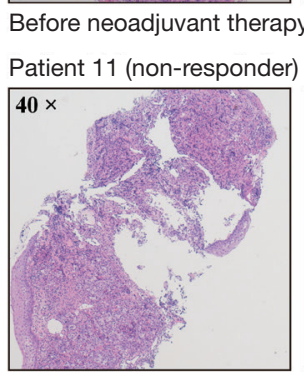

Before neoadjuvant therapy

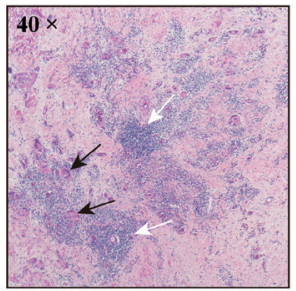

Resected tissue

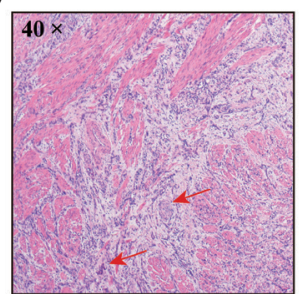

Resected tissue

Figure 1 Clinical and pathological responses to neoadjuvant treatment. (A) Response assessment with CT and surgical pathology. (B) Pathological tumor regression in the resected primary tumor. Green and red denote the presence and absence of lymph node metastases, respectively. The upper and lower dashed lines indicate 50 and 90 regression, respectively. (C) Representative hematoxylin and eosin-stained sections of tumor tissue obtained before neoadjuvant therapy and after surgery (resected tissue) from a patient with pCR (patient 3 ) and a non-responder (patient 11). Black arrows indicate multinucleated giant cells; white arrows indicate tertiary lymphoid structures; red arrows indicate neoplastic cells. Patient 3 and patient 11 were representative of pCR patients and non-responders, respectively. CT, computerized tomography; pCR, complete pathological response. Images are shown at $40 \times$ magnification.

11 tumors evaluable for PD-L1 expression, only two had a CPS of $\geq 10$, one MPR and one non-responder, while all pCR patients had a CPS of $<10$ (Figure $3 A$ ). TMB was also analyzed given its increasing importance for guiding immunotherapy in solid tumors. However, no statistically significant difference in TMB was detected between responders and non-responders. Moreover, the 11 tumors were subjected to $\mathrm{mIF}$ to get a glimpse of their TIME. The densities of CD8+ T cells, TAMs (M1 and M2), and NK cells (CD56 $6^{\text {bright }}$ and CD56 $6^{\mathrm{dim}}$ ) were quantified. CD $56^{\mathrm{dim}} \mathrm{NK}$ cells were significantly more abundant in the responders than in the non-responders $(221.12 \pm 76.83$ vs. $52.33 \pm 11.05$, $\mathrm{P}=0.02$ ) (Figure 3B,3C). Among patients demonstrating $\mathrm{LN}$ down-staging, those who also achieved pCR in the LNs (LN pCR) had significantly more M2-TAMs in the primary tumors than those without LN pCR (LN non-PCR) $(85.29 \pm 21.56$ vs. $437.67 \pm 113.28, \mathrm{P}=0.02$ ) (Figure $3 D, 3 E$ ). No differences were observed in the densities of CD8+ T cells, M1-TAMs, and CD56 $6^{\text {bright }} \mathrm{NK}$ cells between responders and non-responders or between LN pCR and LN non-pCR patients (data not shown).

\section{Discussion}

Our study showed that neoadjuvant treatment of locally advanced ESCC patients with camrelizumab plus nabpaclitaxel and S-1 was well tolerated without causing any grade 3 or higher TRAEs, any surgical delay or any severe perioperative complications. Four patients achieved a pCR and five had a MPR. Radiographic and pathological responses were discrepant for $5 / 12$ cases. Neither PDL1 expression nor TMB was correlated with treatment response and analysis of TIME showed association between the abundance of CD56 ${ }^{\mathrm{dim}} \mathrm{NK}$ cells and M2-TAMs with pathological response in the primary tumors and in the LNs, respectively.

In the preoperative setting, platinum-based chemotherapy plus radiotherapy represents the current standard of care and has been adopted as the combination partner of immunotherapy by a number of ongoing trials $(19,20)$. However, the safety concerns associated with radiotherapy prompted us to seek a less toxic regimen $(12,21,22)$. In lung squamous cell carcinoma, preoperative 

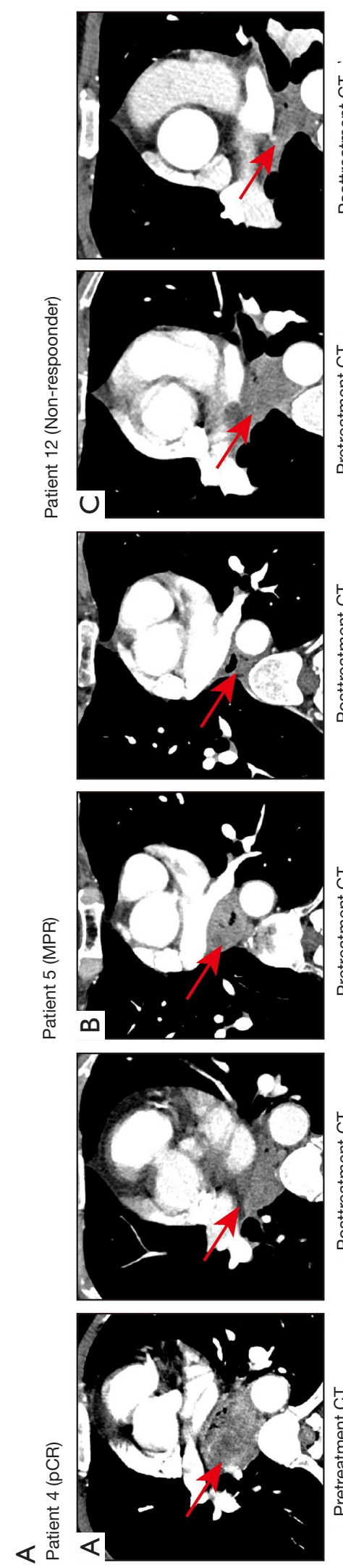
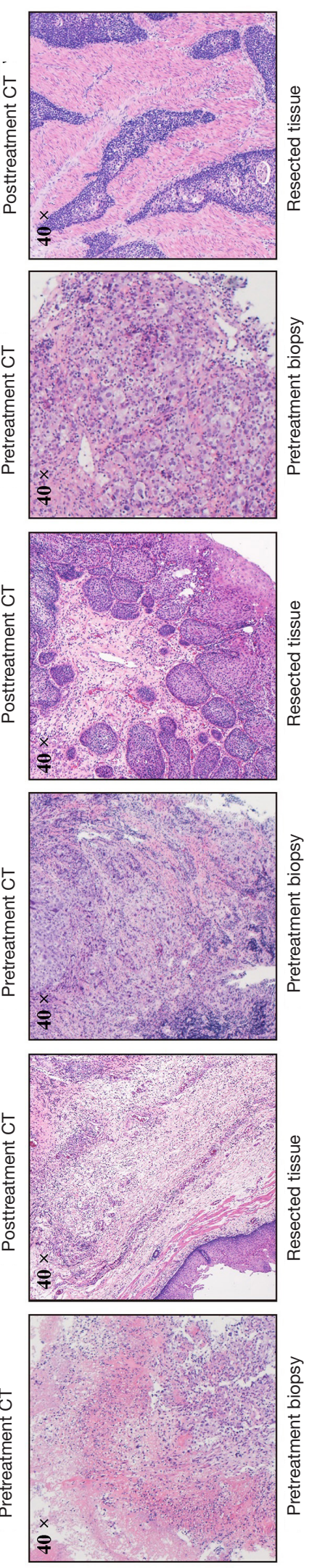
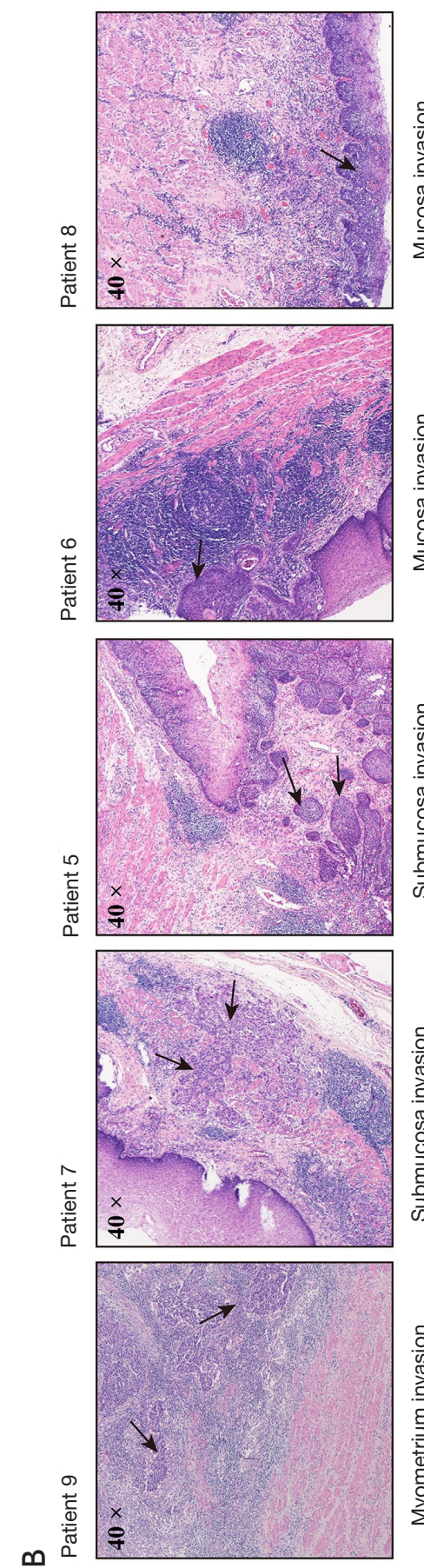

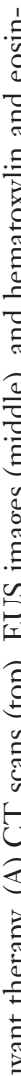

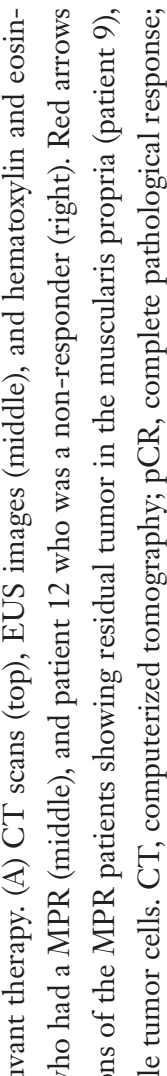

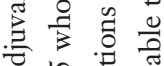

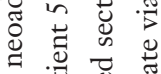

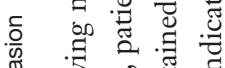

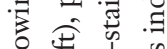

을

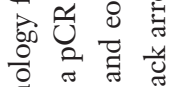

焉

若

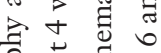

节苞

要

䒕若

. $\widehat{\mathrm{E}}$.

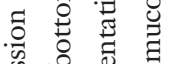

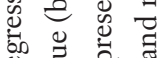

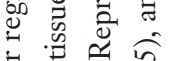

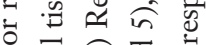

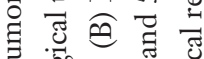

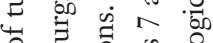

$\infty$ 象

崖

氙 苛 :

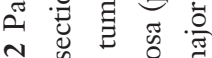

N

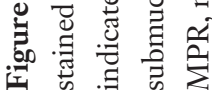


Table 3 Tumor regression evaluated by radiography, endoscopic ultrasonography, and surgical pathology

\begin{tabular}{|c|c|c|}
\hline Patient & Radiographic evaluation & Pathological evaluation \\
\hline \#2 & SD & $\mathrm{pCR}$ \\
\hline \#3 & PR & $\mathrm{pCR}$ \\
\hline \#4 & PR & $\mathrm{pCR}$ \\
\hline \#6 & PR & MPR, residual tumor in mucosa \\
\hline$\# 7$ & PR & MPR, residual tumor in submucosa and mucosa \\
\hline \#8 & SD & MPR, residual tumor in mucosa \\
\hline \#9 & PR & MPR, residual tumor in inner muscularis, submucosa and mucosa \\
\hline$\# 12$ & SD & $\begin{array}{l}\text { Non-responder, residual tumor in adventitia, muscularis, } \\
\text { submucosa, and mucosa }\end{array}$ \\
\hline
\end{tabular}

PR, partial response; SD, stable disease; pCR, complete pathological response; MPR, major pathological response.

atezolizumab combined with nab-paclitaxel and carboplatin was able to induce a $50 \%$ pCR with manageable toxicities (23). Therefore, we were interested to see how ESCC would respond to immunotherapy plus chemotherapy. Indeed, in our study, neoadjuvant administration of nab-paclitaxel plus $\mathrm{S} 1$ in conjunction with camrelizumab, a PD-1 inhibitor approved for second-line treatment of unresectable ESCC in China, demonstrated an excellent safety profile and induced at least $90 \%$ tumor regression in $75 \%$ of the patients. Another study recently presented at ESMO congress 2020 employed a similar regimen comprising nab-paclitaxel/S1 plus toripalimab for preoperative treatment of a similar population, but their pCR rate $(16.67 \%)$ was much lower than ours, which could possibly be explained by the fact that their cohort received only two courses of treatment before surgery while our entire cohort was subjected to 3 cycles of treatment (24).

In another study conducted by Shen et al., the pCR elicited by neoadjuvant PD-1 plus chemotherapy was $33 \%$, which was exactly the same as ours (25). Actually, our study does differ from theirs. A rather homogenous PD-1 inhibitor regimen, camrelizumab was employed in our study. In contrast, in Shen et al.'s study, patients received PD-1 inhibitors such as nivolumab, pembrolizumab, or camrelizumab, which may have elicited different effects.
Secondly, the number of cycles of neoadjuvant treatment in our study was three, which was one more than the two in their research. Several phase 3 trials investigating lung cancer in the neoadjuvant setting have extended the treatment cycle to 3-4 cycles (ClinicalTrials.gov No. NCT03425643, NCT03456063, and NCT03800134). Our results suggest that three cycles of treatment did not increase toxicities of the combinatorial neoadjuvant immune-chemotherapy in locally advanced ESCC.

$\mathrm{LN}$ metastasis is closely associated with poor prognosis in ESCC, but a substantial fraction of patients remains ypN-positive after neoadjuvant treatment, which may later drive postsurgical relapse (26). In our cohort, LN downstaging co-occurred with primary tumor down-staging in nine patients, of which the three pCR patients who had potential baseline LN involvement also achieved pCR in the LNs, indicating that for these patients, incorporation of anti-PD-1 may not only induce anti-tumor immune response in the primary tumor, but also enhance systemic priming of the immune system to eradicate micrometastases in other tissues such as LNs. This notion is also supported by previous evidence in non-small cell lung cancer that some T-cell clones infiltrating pretreatment tumors were also found in the peripheral blood and resected LNs following PD-1 blockade (9). 

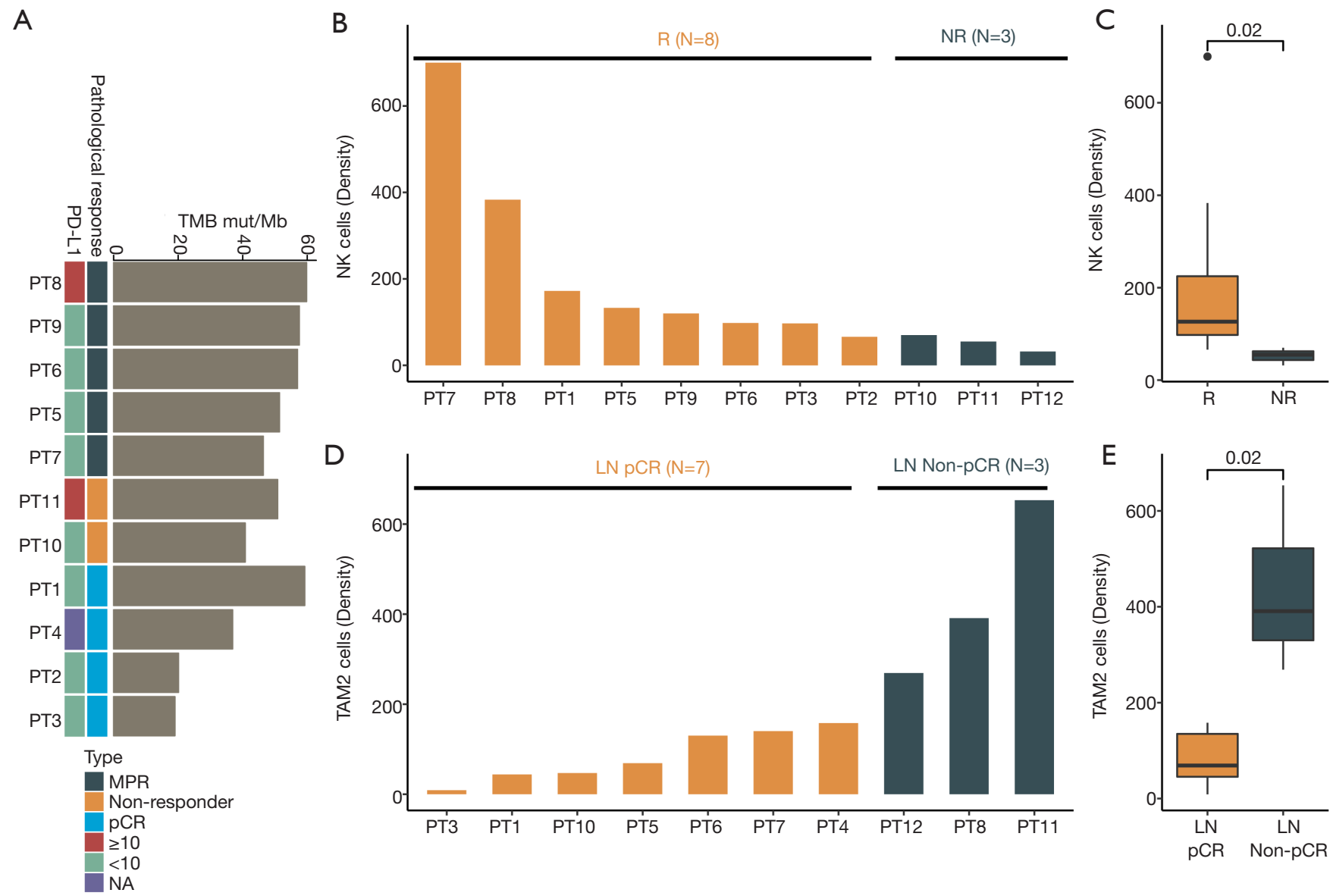

Figure 3 Correlation between TIME and pathological response. (A) Tumor mutational burden and PD-L1 expression among the responders and non-responders as assessed by next generation sequencing with a 733-gene panel and by PD-L1 IHC 22C3 pharmDx assay. $(\mathrm{B}, \mathrm{C})$ Density of natural killer cells in the responders $(\mathrm{R})(221.12 \pm 76.83) v s$. the NR $(52.33 \pm 11.05)(\mathrm{P}=0.02$ by exact Wilcoxon test). (D,E) Density of M2-like tumor-associated macrophages in the patients with complete pathological response in the LNs (pCR) (437.67 \pm 113.28 ) $v s$. patients without complete pathological response in the lymph nodes $(85.29 \pm 21.56)(\mathrm{P}=0.02$ by exact Wilcoxon test). Density was defined as the number of stained cells per square millimeter. TIME, tumor immune microenvironment; PD-L1, programmed death-ligand 1; NR, non-responders; LN, lymph node; pCR, complete pathological response.

Despite the approval of pembrolizumab in advanced esophageal cancer with a CPS PD-L1 of $\geq 10$ and in advanced solid tumors with a TMB of $\geq 10$ mutations/ megabase, the roles of PD-L1 and TMB as predictors of immune checkpoint blockade remained to be clarified (20). Indeed, our observations showed poor correlation of PD-L1 expression or TMB with pathological response to neoadjuvant immunochemotherapy. It is therefore important to explore other biomarkers to guide patient selection. Consistent with previous literature showing positive correlation between NK cell abundance and clinical response to nivolumab in advanced melanoma,
NK cells were found at a higher density in the responders in our cohort (27). Moreover, these NK cells belonged to the $\mathrm{CD} 56^{\mathrm{dim}}$ subset, the mature form of NK cells with more potent antitumor effects, which may help explain the favorable response in the responders (28). In addition, M2TAMs were more abundant in patients who remained LNpositive after neoadjuvant treatment. This is supported by the fact that M2-TAMs may subvert immune surveillance by producing immune-suppressing cytokines, rendering the patients resistant to immunotherapy (29).

Our study was limited by its retrospective design and a small sample size. However, we included a rather 


\section{Page 10 of 11}

homogenous population where treatment regimen and schedule were both consistent across the entire cohort, which to some extent ensured data quality. Secondly, the follow-up time was too short for us to fully assess the impact of favorable pathological responses on recurrence-free and overall survivals. A larger phase II study with long-term follow-up is currently underway to confirm our findings in a prospective manner (ChiCTR2000029807). Taken together, neoadjuvant administration of PD-1 blockade in combination with chemotherapy was tolerable, feasible, and efficacious in locally advanced ESCC.

\section{Acknowledgments}

The authors would like to acknowledge all patients participating in the study.

Funding: This study was supported by the Science and Technology Planning Project of Guangdong Province of China (2017A020215179) and the Science and Technology Planning Project of Guangzhou of China (202103000064).

\section{Footnote}

Reporting Checklist: The authors have completed the STROBE reporting checklist. Available at https://dx.doi. org/10.21037/atm-21-3352

Data Sharing Statement: Available at https://dx.doi. org/10.21037/atm-21-3352

Conflicts of Interest: All authors have completed the ICMJE uniform disclosure form (available at https://dx.doi. org/10.21037/atm-21-3352). Dr. CG, YZ, WX, MH, TB, and $\mathrm{YB}$ are employees of 3D Medicines Inc. The other authors have no other conflicts of interest to declare.

Ethical Statement: The authors are accountable for all aspects of the work in ensuring that questions related to the accuracy or integrity of any part of the work are appropriately investigated and resolved. This study was approved by the institutional review boards at Sun Yatsen University Cancer Center (B2020-292-01) and all patients provided written informed consents. All procedures performed in this study involving human participants were in accordance with the Declaration of Helsinki (as revised in 2013).

Open Access Statement: This is an Open Access article
Yang et al. Neoadjuvant Anti-PD-1 plus Chemotherapy in ESCC

distributed in accordance with the Creative Commons Attribution-NonCommercial-NoDerivs 4.0 International License (CC BY-NC-ND 4.0), which permits the noncommercial replication and distribution of the article with the strict proviso that no changes or edits are made and the original work is properly cited (including links to both the formal publication through the relevant DOI and the license). See: https://creativecommons.org/licenses/by-nc-nd/4.0/.

\section{References}

1. Bray F, Ferlay J, Soerjomataram I, et al. Global cancer statistics 2018: GLOBOCAN estimates of incidence and mortality worldwide for 36 cancers in 185 countries. CA Cancer J Clin 2018;68:394-424.

2. Abnet CC, Arnold M, Wei WQ. Epidemiology of Esophageal Squamous Cell Carcinoma. Gastroenterology 2018;154:360-73.

3. Kong M, Shen J, Zhou C, et al. Prognostic factors for survival in esophageal squamous cell carcinoma (ESCC) patients with a complete regression of the primary tumor (ypT0) after neoadjuvant chemoradiotherapy (NCRT) followed by surgery. Ann Transl Med 2020;8:1129.

4. Xi M, Yang Y, Zhang L, et al. Multi-institutional Analysis of Recurrence and Survival After Neoadjuvant Chemoradiotherapy of Esophageal Cancer: Impact of Histology on Recurrence Patterns and Outcomes. Ann Surg 2019;269:663-70.

5. Deng J, Xia Y, Chen Y, et al. Long term results of different radiotherapy techniques and fractions for esophageal squamous cell carcinoma. Transl Cancer Res 2020;9:2287-94.

6. Liedman B, Johnsson E, Merke C, et al. Preoperative adjuvant radiochemotherapy may increase the risk in patients undergoing thoracoabdominal esophageal resections. Dig Surg 2001;18:169-75.

7. Kato K, Cho BC, Takahashi M, et al. Nivolumab versus chemotherapy in patients with advanced oesophageal squamous cell carcinoma refractory or intolerant to previous chemotherapy (ATTRACTION-3): a multicentre, randomised, open-label, phase 3 trial. Lancet Oncol 2019;20:1506-17.

8. Shah MA, Adenis A, Enzinger PC, et al. Pembrolizumab versus chemotherapy as second-line therapy for advanced esophageal cancer: Phase 3 KEYNOTE-181 study. J Clin Oncol 2019;37:abstr 4010.

9. Liang W, Cai K, Chen C, et al. Expert consensus on neoadjuvant immunotherapy for non-small cell lung 
cancer. Transl Lung Cancer Res 2020;9:2696-715.

10. Chalabi M, Fanchi LF, Dijkstra KK, et al. Neoadjuvant immunotherapy leads to pathological responses in MMRproficient and MMR-deficient early-stage colon cancers. Nat Med 2020;26:566-76.

11. Amaria RN, Reddy SM, Tawbi HA, et al. Neoadjuvant immune checkpoint blockade in high-risk resectable melanoma. Nat Med 2018;24:1649-54.

12. Lee S, Ahn BC, Park SY, et al. A phase II trial of preoperative chemoradiotherapy and pembrolizumab for locally advanced esophageal squamous cell carcinoma (ESCC). J Clin Oncol 2019;37:abstr 4027.

13. National Cancer Institute Common Terminology Criteria for Adverse Events (CTCAE), version 5.0 for adverse event reporting 2019.

14. Eisenhauer EA, Therasse P, Bogaerts J, et al. New response evaluation criteria in solid tumours: revised RECIST guideline (version 1.1). Eur J Cancer 2009;45:228-47.

15. Su D, Zhang D, Chen K, et al. High performance of targeted next generation sequencing on variance detection in clinical tumor specimens in comparison with current conventional methods. J Exp Clin Cancer Res 2017;36:121.

16. Cabrita R, Lauss M, Sanna A, et al. Tertiary lymphoid structures improve immunotherapy and survival in melanoma. Nature 2020;577:561-5.

17. Helmink BA, Reddy SM, Gao J, et al. B cells and tertiary lymphoid structures promote immunotherapy response. Nature 2020;577:549-55.

18. Gao J, Navai N, Alhalabi O, et al. Neoadjuvant PD-L1 plus CTLA-4 blockade in patients with cisplatin-ineligible operable high-risk urothelial carcinoma. Nat Med 2020;26:1845-51.

19. Ajani JA, D'Amico TA, Bentrem DJ, et al. Esophageal and Esophagogastric Junction Cancers, Version 2.2019, NCCN Clinical Practice Guidelines in Oncology. J Natl Compr Canc Netw 2019;17:855-83.

20. Vivaldi C, Catanese S, Massa V, et al. Immune Checkpoint
Inhibitors in Esophageal Cancers: are we Finally Finding the Right Path in the Mist? Int J Mol Sci 2020;21:1658.

21. Hartmann JT, Lipp HP. Toxicity of platinum compounds. Expert Opin Pharmacother 2003;4:889-901.

22. Giuranno L, Ient J, De Ruysscher D, et al. RadiationInduced Lung Injury (RILI). Front Oncol 2019;9:877.

23. Shu CA, Gainor JF, Awad MM, et al. Neoadjuvant atezolizumab and chemotherapy in patients with resectable non-small-cell lung cancer: an open-label, multicentre, single-arm, phase 2 trial. Lancet Oncol 2020;21:786-95.

24. Zhang G, Hu Y, Yang B, et al. A single-centre, prospective, open-label, single-arm trial of toripalimab with nab-paclitaxel and S-1 as a neoadjuvant therapy for esophageal squamous cell carcinoma (ESCC). Ann Oncol 2020;31:S645-71.

25. Shen $\mathrm{D}, \mathrm{Chen} \mathrm{Q}, \mathrm{Wu} \mathrm{J}$, et al. The safety and efficacy of neoadjuvant PD-1 inhibitor with chemotherapy for locally advanced esophageal squamous cell carcinoma. J Gastrointest Oncol 2021;12:1-10.

26. Leng X, He W, Yang H, et al. Prognostic Impact of Postoperative Lymph Node Metastases After Neoadjuvant Chemoradiotherapy for Locally Advanced Squamous Cell Carcinoma of Esophagus: From the Results of NEOCRTEC5010, a Randomized Multicenter Study. Ann Surg 2019. [Epub ahead of print]. doi: 10.1097/ SLA.0000000000003727.

27. Riaz N, Havel JJ, Makarov V, et al. Tumor and Microenvironment Evolution during Immunotherapy with Nivolumab. Cell 2017;171:934-949.e16.

28. Wagner JA, Rosario M, Romee R, et al. CD56bright NK cells exhibit potent antitumor responses following IL-15 priming. J Clin Invest 2017;127:4042-58.

29. Li X, Liu R, Su X, et al. Harnessing tumor-associated macrophages as aids for cancer immunotherapy. Mol Cancer 2019;18:177.

(English Language Editor: G. Stone)
Cite this article as: Yang G, Su X, Yang H, Luo G, Gao C, Zheng Y, Xie W, Huang M, Bei T, Bai Y, Wang Z, Cai P, He H, Xiang J, Cai M, Zhang Y, Qu C, Fu J, Liu Q, Hu Y, Zhong J, Huang Y, Guo Q, Zhang X. Neoadjuvant programmed death-1 blockade plus chemotherapy in locally advanced esophageal squamous cell carcinoma. Ann Transl Med 2021;9(15):1254. doi: $10.21037 / \mathrm{atm}-21-3352$ 
Table S1 List of genes of the 733-gene panel

\begin{tabular}{|c|c|c|c|c|c|c|c|c|c|c|c|c|c|c|}
\hline ABL1 & CDX2 & FGFR4 & MLH1 & PTEN & VEGFA & JMJD1C & TRIM37 & BCL11A & EZR & TBL1XR1 & PLXNB1 & LIG1 & RNF168 & POLD3 \\
\hline ACVR2A & $\mathrm{CHD} 2$ & $\mathrm{FH}$ & MLLT3 & PTK6 & VHL & LMO1 & TSHR & BCL11B & FAT4 & TCF7L2 & SPRED1 & LIG3 & RNF4 & POLD4 \\
\hline AFF3 & CHEK1 & FHIT & MPL & PTPRD & NSD3 & LZTR1 & UROD & BCORL1 & FUBP1 & TCL1A & ERF & LIG4 & RNF8 & POLE2 \\
\hline AKT1 & CHEK2 & FLCN & MRE11A & RAC1 & ZNF479 & MAX & WAS & BIRC3 & FUS & TET1 & RPS6KA3 & MAD2L2 & RPA1 & POLE4 \\
\hline AKT2 & $\mathrm{CHIC2}$ & FLT1 & MSH2 & RAD50 & ZNRF3 & MEN1 & WRN & BRD4 & GAS7 & TFE3 & GSK3B & MBD4 & RPA2 & PPP4R1 \\
\hline AKT3 & $\mathrm{CIC}$ & FLT3 & $\mathrm{MSH} 3$ & RAD51 & ABCB11 & MTAP & WT1 & CACNA1D & H3F3A & TNFAIP3 & NOTCH3 & MDC1 & RPA3 & PPP4R3A \\
\hline ALK & CIITA & FLT4 & MSH6 & RAD51C & АРОВЕС3В & MUTYH & XPA & CALR & HIF1A & USP8 & $\mathrm{NOTCH} 4$ & MGMT & RPA4 & PPP4R3B \\
\hline ANK1 & CRBN & FOXA1 & MTOR & RAF1 & AXIN2 & NBN & XPC & CAMTA1 & HIP1 & WIF1 & ALKBH2 & MLH3 & RRM2B & PPP4R4 \\
\hline APC & CRLF2 & FRS2 & MYC & RARA & BARD1 & NHP2 & XRCC2 & CANT1 & HNRNPA2B1 & XPO1 & ALKBH3 & MMS19 & SETMAR & RAD9B \\
\hline AR & CRNKL1 & G6PD & MYCN & RB1 & BMPR1A & NME1 & HOXB13 & CARD11 & HOXA11 & ZFHX3 & APEX1 & MNAT1 & SEM1 & RBX1 \\
\hline ARAF & CRTC3 & GATA3 & MYD88 & RET & BUB1B & NOP10 & BCL2L1 & KNL1 & IL6ST & ACVR1B & APEX2 & MPG & SHPRH & RFC1 \\
\hline AREG & CSF1R & GLI2 & NF1 & RGS7 & $\mathrm{CDC73}$ & NTHL1 & BCL6 & CASP8 & KDM6A & ARID1B & CENPS & $\mathrm{MSH} 4$ & SMUG1 & RFC2 \\
\hline ARHGAP5 & CSF3R & GNA11 & NF2 & RICTOR & CDKN1C & PHOX2B & CDK8 & CBFA2T3 & KEAP1 & DNMT1 & APLF & MUS81 & SPO11 & RFC3 \\
\hline ARID1A & CTNNB1 & GNAQ & NFE2L2 & RNF43 & CEBPA & PMS1 & FOXP1 & CBFB & KLF4 & FOXL2 & APTX & NEIL1 & TDG & RFC4 \\
\hline ARNT & CTNND2 & GNAS & NFIB & ROS1 & COL7A1 & POLH & GRIN2A & CBLB & LCK & GATA1 & ATRIP & NEIL2 & TDP1 & TELO2 \\
\hline ASXL1 & CUL3 & HDAC2 & NKX2-1 & RPTOR & CTR9 & POLQ & IKBKE & CCDC6 & LEF1 & HIST1H3B & FAAP100 & NEIL3 & TDP2 & TIMELESS \\
\hline ATM & CYSLTR2 & HEY1 & NOTCH1 & RUNX1 & CXCR4 & POT1 & MEF2B & CCNB1IP1 & LIFR & KDM5C & FAAP24 & NHEJ1 & TOP3A & TMEM189 \\
\hline ATR & DDR2 & $\mathrm{HGF}$ & NOTCH2 & SDC4 & CYLD & PRDM9 & NFKBIA & CD79A & MAPK1 & MAP3K1 & FAAP20 & NUDT1 & TOP3В & WDR48 \\
\hline AURKA & DICER1 & HOOK3 & NPM1 & SDHC & DDB2 & PRF1 & PIK3CD & CD79B & MED12 & KMT2C & MPLKIP & NABP2 & TOPBP1 & GFI1 \\
\hline$A X L$ & DNMT3A & HRAS & NRAS & SERPINB3 & DIS3L2 & PRKAR1A & $\mathrm{SRC}$ & $\mathrm{CDH} 11$ & NAB2 & NCOR1 & $\mathrm{CCNH}$ & OGG1 & TP53BP1 & CYP17A1 \\
\hline B2M & DPYD & IDH1 & NRG1 & SETD2 & DKC1 & PRSS1 & BTG1 & CHD4 & NCOR2 & PHF6 & CDK7 & PARP1 & TREX1 & ELF3 \\
\hline BAP1 & EGFR & IDH2 & NTRK1 & SF3B1 & DOCK8 & PTPN11 & DIS3 & CLIP1 & NDRG1 & PPP2R1A & CETN2 & PARP2 & TREX2 & SGK1 \\
\hline BAZ1A & EPHA2 & IGF1R & NTRK2 & SH2B3 & DROSHA & PTPN13 & EED & CLTCL1 & NONO & PRDM1 & CHAF1A & PARP3 & UBE2A & GSTT1 \\
\hline BCL2 & EPHA3 & IGF2 & NTRK3 & SLC29A1 & ELANE & RAD51B & GNA13 & CNBP & PAX3 & socs1 & CLK2 & PCNA & UBE2B & AEN \\
\hline BCOR & ERBB2 & IL7R & PAK1 & SMAD4 & EPCAM & RAD51D & NT5C2 & CNOT3 & PAX7 & sox9 & DCLRE1A & PNKP & UBE2N & CCNO \\
\hline BLM & ERBB3 & INPP4B & PALB2 & SMARCA1 & ERCC3 & RECQL & PPP2R2A & CREB3L1 & PAX8 & TRAF7 & DCLRE1B & POLB & UBE2T & CENPX \\
\hline BMP5 & ERBB4 & ITGAV & PAX5 & SMARCA4 & ERCC5 & RECQL4 & NSD2 & CREB3L2 & PER1 & IKZF1 & DCLRE1C & POLI & UBE2V2 & CUL4A \\
\hline BRAF & ERCC1 & JAK1 & PBRM1 & SMARCB1 & ETV6 & RFWD3 & EPHA7 & CREBBP & PICALM & MYCL & DDB1 & POLK & UNG & CUL5 \\
\hline BRCA1 & ERCC2 & JAK2 & PDCD1LG2 & SMO & EXT1 & RHBDF2 & GLI1 & CRTC1 & PIM1 & NCOA3 & $\mathrm{DMC} 1$ & POLL & USP1 & DNTT \\
\hline BRCA2 & ERCC4 & JAK3 & PDGFB & SRGAP3 & EXT2 & SBDS & MYB & CTCF & POU2AF1 & CDK2 & DUT & POLM & XАB2 & ELOA \\
\hline BRIP1 & ERCC6 & JUN & PDGFRA & SRSF2 & $\mathrm{FAH}$ & SDHA & NRG3 & CUX1 & POU5F1 & LATS1 & EME1 & POLN & XRCC1 & HUS1B \\
\hline BTK & EREG & KCNJ5 & PDGFRB & STAG2 & FANCD2 & SDHAF2 & NUP93 & DAXX & PPP6C & LATS2 & EME2 & PRKDC & XRCC3 & PER2 \\
\hline CARS & ESR1 & $\mathrm{KDR}$ & PDPK1 & STK11 & FANCE & SDHB & PTK2 & DDIT3 & PRDM16 & YAP1 & ENDOV & PRPF19 & XRCC4 & PER3 \\
\hline $\mathrm{CBL}$ & EWSR1 & KIT & PIK3CA & SUZ12 & FANCF & SDHD & RXRA & DDX10 & PREX2 & TEAD2 & ERCC8 & RAD1 & XRCC5 & MSH5 \\
\hline CCND1 & $\mathrm{EZH} 2$ & KMT2A & PIK3CB & SYK & FANCI & SERPINA1 & SMARCA2 & DDX3X & PRKACA & MGA & EXO1 & RAD18 & XRCC6 & PARP4 \\
\hline CCND2 & FAM135B & KMT2D & PIK3R1 & TBX3 & FANCL & SETBP1 & TYK2 & DDX5 & PTPRT & HES1 & FAN1 & RAD23A & ABRAXAS1 & POLE3 \\
\hline CCND3 & FAM47C & KRAS & PIK3R2 & TCF3 & FANCM & SH2D1A & ZNF750 & DDX6 & QKI & KDM5A & FANCB & RAD23B & FRK & PPP4R2 \\
\hline CCNE1 & FANCA & LASP1 & PLCG2 & TERT & FAS & $\mathrm{SHOC2}$ & $\mathrm{ABI} 1$ & DNM2 & RAD21 & SPEN & GEN1 & RAD52 & BIRC5 & SLX1A \\
\hline CD274 & FANCC & LMNA & PML & TET2 & FEN1 & SLC25A13 & ACKR3 & EBF1 & RANBP2 & THBS2 & GTF2H1 & RAD54B & EMSY & RAD54L2 \\
\hline $\mathrm{CDH} 1$ & FANCG & LRP1B & PMS2 & TMEM127 & GALNT12 & SLX4 & ACSL3 & EIF3E & RAP1GDS1 & CUL1 & GTF2H3 & RAD54L & CRKL & RFC5 \\
\hline $\mathrm{CDH} 10$ & FAT1 & MAP2K1 & POLD1 & TMPRSS2 & GATA2 & sos1 & ACVR1 & EIF4A2 & RBM10 & HDAC1 & GTF2H4 & RAD9A & EPHB1 & HMGA2 \\
\hline CDK12 & FBXW7 & MAP2K2 & POLE & TOP2A & GBA & SPOP & AFF4 & ELF4 & $\mathrm{RHOA}$ & MLST8 & GTF2H5 & RBBP8 & GLI3 & TSPAN31 \\
\hline CDK4 & FES & MAP2K4 & POLG & TP53 & GJB2 & SPRTN & AMER1 & ELK4 & $\mathrm{RHOH}$ & PIK3R3 & H2AFX & RDM1 & IRS2 & MYOD1 \\
\hline CDK6 & FGF19 & MCL1 & PPARG & TPMT & GPC3 & SRY & ARID2 & ELL & RNF213 & RHEB & HELQ & RECQL5 & RUNX1T1 & CHD1 \\
\hline CDKN1A & FGF3 & MDM2 & PPM1D & TSC1 & GREM1 & STAT3 & ATP1A1 & EP300 & SFPQ & RPS6KB1 & HFM1 & REV1 & SLIT2 & ZBTB16 \\
\hline CDKN1B & FGF4 & MDM4 & PRCC & TSC2 & HFE & SUFU & ATP2B3 & EPAS1 & SLC34A2 & GRB2 & HLTF & REV3L & sox2 & PCDH9 \\
\hline CDKN2A & FGFR1 & MECOM & PRKCH & U2AF1 & HMBS & TGFBR1 & ATRX & EPS15 & SLC45A3 & RIT1 & HMGB1 & RIF1 & SPTA1 & PLXNA1 \\
\hline CDKN2B & FGFR2 & MET & PSIP1 & UGT1A1 & HNF1A & TGFBR2 & AXIN1 & ERC1 & SMAD2 & RASA1 & HUS1 & RMI1 & ZNF217 & \\
\hline CDKN2C & FGFR3 & MITF & PTCH1 & USP6 & ITK & TP63 & BCL10 & ETNK1 & SMAD3 & ERRFI1 & UVSSA & RMI2 & ZNF703 & \\
\hline
\end{tabular}


Table S2 Clinicophysiological characteristics of the patients

\begin{tabular}{|c|c|c|c|c|c|c|c|c|c|c|c|c|c|c|}
\hline No. & Gender & Age & Smoking $^{a}$ & Drinking $^{a}$ & $\begin{array}{l}\text { Site of primary } \\
\text { tumor }\end{array}$ & Differentiation & cTNM $^{e}$ & $\begin{array}{l}\text { Clinical } \\
\text { response }^{c}\end{array}$ & pTNM $^{e}$ & $\begin{array}{l}\text { Pathological } \\
\text { response }^{f}\end{array}$ & T downstage & $\mathrm{N}$ downstage & $\begin{array}{l}\text { No. of } \\
\text { LND }^{\mathrm{b}}\end{array}$ & $\begin{array}{l}\text { No. of } \\
\text { LNM }^{d}\end{array}$ \\
\hline 1 & Male & 56 & $\mathrm{Y}$ & $\mathrm{Y}$ & Middle & Well & $\begin{array}{l}\text { cT2N2MO } \\
\text { III }\end{array}$ & PR & pTONOMO & pCR & $\mathrm{Y}$ & $\mathrm{Y}$ & 34 & 0 \\
\hline 2 & Female & 60 & $\mathrm{~N}$ & $\mathrm{~N}$ & Middle & Moderately & cT3NOMO II & SD & pTONOMO & $\mathrm{pCR}$ & Y & - & 26 & 0 \\
\hline 3 & Female & 65 & $\mathrm{~N}$ & $\mathrm{~N}$ & Middle & Poorly & cT3N1MO III & PR & pTONOMO & $\mathrm{pCR}$ & $\mathrm{Y}$ & Y & 34 & 0 \\
\hline 4 & Male & 56 & $\mathrm{~N}$ & $\mathrm{~N}$ & Lower & Poorly & cT3N1M0 III & PR & pTONOMO & $\mathrm{pCR}$ & Y & $\mathrm{Y}$ & 24 & 0 \\
\hline 5 & Male & 51 & $\mathrm{Y}$ & $\mathrm{Y}$ & Lower & Poorly & cT3N1M0 III & PR & pT1bNOMO & MPR & $\mathrm{Y}$ & $\mathrm{Y}$ & 30 & 0 \\
\hline 6 & Male & 51 & $\mathrm{Y}$ & $\mathrm{Y}$ & Upper & Moderately & cT2N2MO III & PR & pTisNOMO & MPR & $\mathrm{Y}$ & $\mathrm{Y}$ & 28 & 0 \\
\hline 7 & Male & 63 & $\mathrm{Y}$ & Y & Lower & Poorly & cT2N1MO II & PR & pT1bNOMO & MPR & $\mathrm{Y}$ & Y & 63 & 0 \\
\hline 8 & Male & 50 & $\mathrm{Y}$ & Y & Middle & Moderately & cT3N3M0 IVA & SD & pTisN1M0 & MPR & $\mathrm{Y}$ & $\mathrm{Y}$ & 50 & 1 \\
\hline 9 & Male & 56 & $\mathrm{Y}$ & $\mathrm{Y}$ & Lower & Poorly & cT3N3MO IVA & PR & pT2N2Mo & MPR & $\mathrm{Y}$ & $\mathrm{Y}$ & 54 & 3 \\
\hline 10 & Female & 62 & $\mathrm{Y}$ & Y & Lower & Poorly & cT3N2MO III & SD & pT2NOMO & Non-responder & $\mathrm{Y}$ & $\mathrm{Y}$ & 35 & 0 \\
\hline 11 & Female & 63 & $\mathrm{~N}$ & $\mathrm{~N}$ & Middle & Poorly & cT3N1M0 III & SD & pT3N1M0 & Non-responder & $N$ & $\mathrm{~N}$ & 27 & 1 \\
\hline 12 & Female & 51 & $\mathrm{~N}$ & $\mathrm{~N}$ & Middle & Poorly & cT3N2MO III & SD & pT3N2M0 & Non-responder & $\mathrm{N}$ & $\mathrm{N}$ & 61 & 4 \\
\hline
\end{tabular}

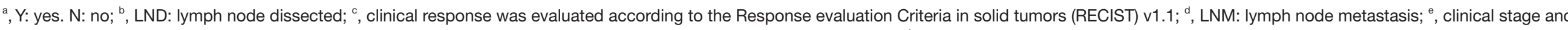

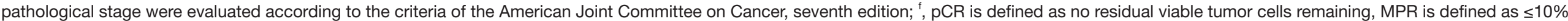
viable tumor cells remaining, non-responder is defined as $\geq 50 \%$ residual viable tumor cells. 